\title{
A New Fuzzy Information Inequalities and its Applications in Establishing Relation among Fuzzy $f$-Divergence Measures
}

\author{
Sapna Gahlot and Ram Naresh Saraswat
}

\begin{abstract}
There are many fuzzy information and divergence measures exist in the literature of fuzzy Information Theory. Inequalities play important role for finding the relations. Here, we will introduce some new information inequalities on fuzzy measures and their applications in pattern recognition. Also established relations between new and well known fuzzy divergence measures with help of the fuzzy $f$-divergence measures and jensen's inequality.
\end{abstract}

\section{Introduction}

In information theory, Shannon [28] defined the entropy measure for a probability distribution. Fuzzy entropy, a fuzziness measure (cf. [21]) often used and cited in many literatures, was introduced by Zadeh [34, 35]; then De Luca and Termini [20] defined an entropy of a fuzzy set based on Shannon's function. Later on, many other researchers made more effort in this particular area. In 1975, Kaufmann [18] proposed a new fuzziness measure of fuzzy set by a distance between its membership function and its nearest Classical(Ordinary) sets. It has broad applications in many areas such as Pattern recognition, image processing, fuzzy clustering, speech recognition, feature selection, fuzzy aircraft control, bio-informatics etc.

Afterwards, a number of other researchers have studied the fuzzy divergence measures in different ways [26] and provide their applications in different areas. The fuzzy divergence measure introduced by Fan and Xie [8] is based on exponential operation and its relation with fuzzy divergence measures [2]. Prakash et al. [23] proposed two fuzzy divergence measures corresponding to Ferreri [11] probabilistic measure of directed divergence. Ghosh et al. [14] gave its application in the area of automated leukocyte recognition. The study submitted by Montes et al. [22] in 2002 was based on special classes of divergence measures and used the link between fuzzy and probabilistic uncertainty. Bhatia and Singh [4] proposed the fuzzy divergence measure corresponding to Taneja [30]. Tomar and Ohlan [32] studied a sequence of fuzzy mean difference divergence 2010 Mathematics Subject Classification. 58F15, 58F17, 53C35.

Key words and phrases. Fuzzy set, fuzzy information measures, fuzzy $f$-divergence measures, Jensen's inequality, fuzzy inequalities..

Corresponding author: Ram Naresh Saraswat. 
measures by establishing inequalities among them and provided their applications in the context of consistency in linguistic variables and pattern recognition.

Entropy is the important concept of Information Theory. Shannon [28] use the entropy to measure a degree of uncertainty of randomness in a p.d. Let

$$
\Gamma_{n}=\left\{P=\left(p_{1}, p_{2}, p_{3}, \cdots, p_{n}\right): p_{i} \geq 0, \sum_{i=1}^{n} p_{i}=1,\right\}
$$

be a set of complete finite discrete p.d., and

$$
H(P)=\sum_{i=1}^{n}-p_{i} \log p_{i}
$$

Directed divergence is a cross entropy measure which provides a distance between the two p.d. Kullback and Leibler [19] proposed a measure of directed divergence between the two distributions $P=\left(p_{1}, p_{2}, \cdots, p_{n}\right)$ and $Q=\left(q_{1}, q_{2}, \cdots, q_{n}\right)$ as:

$$
D(P \mid Q)=\sum_{i=1}^{n} p_{i} \log \left(\frac{p_{i}}{q_{i}}\right)
$$

The Csiszar's $f$-divergence measure and new $f$-divergence measure contains divergences used in determining the affinity between two p.d using a convex function $f$, defined on $(0, \infty)$.

$$
C_{f}(P, Q)=\sum_{i=1}^{n} q_{i} f\left(\frac{p_{i}}{q_{i}}\right)
$$

and

$$
S_{f}(P, Q)=\sum_{i=1}^{n} q_{i} f\left(\frac{p_{i}+q_{i}}{2 q_{i}}\right) .
$$

\section{Fuzzy Sets and Fuzzy Measures of Information}

Fuzziness is a feature of uncertainty, results from the lack of sharp difference of being or not being a member of the set. A fuzzy set $A$ defined on a universe of discourse $X$ is given as Zadeh [34]

$$
A=\left\{\left\langle x, \mu_{A}(x) / x \in X\right\rangle\right\}
$$

where $\mu_{A}(x): X \rightarrow[0,1]$ is the membership function of $A$. The membership value describes the degree of belongingness of $X$ in $A$.

Fuzzy entropy corresponding to Shannon's entropy given as 


$$
H(N)=\sum_{i=1}^{n}-\left(\mu_{A}\left(x_{i}\right) \log \mu_{A}\left(x_{i}\right)+\left(1-\mu_{A}\left(x_{i}\right)\right) \log \left(1-\mu_{A}\left(x_{i}\right)\right)\right) .
$$

This idea of divergence measure was extended from probabilistic to fuzzy set theory by giving a fuzzy information measure for discrimination of a fuzzy set B relative to some other fuzzy set $A$. Let $A$ and $B$ be two fuzzy sets defined in universe of discourse $X=\left\{x_{1}, x_{2}, \cdots, x_{n}\right\}$ having the membership values $\mu_{A}\left(x_{i}\right)$ and $\mu_{B}\left(x_{i}\right), i=1,2, \cdots, n$ respectively. Then the fuzzy divergence measures : (Here $\mu_{A}\left(x_{i}\right)$ and $\mu_{B}\left(x_{i}\right)$ are denoted by $\mu_{A_{i}}$ and $\mu_{B_{i}}$.)

(i) Fuzzy K-L Divergence [19]-

$$
K(A, B)=\sum_{i=1}^{n}\left[\mu_{A_{i}} \log \left(\frac{\mu_{A_{i}}}{\mu_{B_{i}}}\right)+\left(1-\mu_{A_{i}}\right) \log \left(\frac{1-\mu_{A_{i}}}{1-\mu_{B_{i}}}\right)\right]
$$

(ii) Fuzzy Relative Information of type n [31] :-

$$
D_{n}(A, B)=(n(n-1))^{-1}\left[\sum_{i=1}^{n}\left(\left(\mu_{A_{i}}\right)^{n}\left(\mu_{B_{i}}\right)^{1-n}+\left(1-\mu_{A_{i}}\right)^{n}\left(1-\mu_{B_{i}}\right)^{1-n}\right)-1\right],
$$

where $n \neq 0,1$.

(iii) Fuzzy Chi-Square Divergence [24]:-

$$
\chi^{2}(A, B)=\sum_{i=1}^{n}\left[\frac{\left(\mu_{A_{i}}\right)^{2}}{\mu_{B_{i}}}+\frac{\left(1-\mu_{A_{i}}\right)^{2}}{1-\mu_{B_{i}}}\right]-1,
$$

(iv) Fuzzy Relative Jensen-Shannon Divergence [33]:-

$$
S_{t}(A, B)=\sum_{i=1}^{n}\left[\mu_{A_{i}} \log \left(\frac{2 \mu_{A_{i}}}{\mu_{A_{i}}+\mu_{B_{i}}}\right)+\left(1-\mu_{A_{i}}\right) \log \left(\frac{2\left(1-\mu_{A_{i}}\right)}{2-\mu_{A_{i}}-\mu_{B_{i}}}\right)\right]
$$

(v) Fuzzy Relative Arithmetic-Geometric Divergence [5]:-

$$
\begin{aligned}
H(A, B)=\sum_{i=1}^{n}[ & \left(\frac{\mu_{A_{i}}+\mu_{B_{i}}}{2}\right) \log \left(\frac{\mu_{A_{i}}+\mu_{B_{i}}}{2 \mu_{A_{i}}}\right)+ \\
& \left.\left(\frac{2-\mu_{A_{i}}-\mu_{B_{i}}}{2}\right) \log \left(\frac{2-\mu_{A_{i}}-\mu_{B_{i}}}{2\left(1-\mu_{A_{i}}\right)}\right)\right]
\end{aligned}
$$

(vi) Fuzzy Unified Relative Jensen-Shannon and Arithmetic-Geometric Divergence of type $n$

$$
M G_{n}(A, B)=(n(n-1))^{-1}\left(\sum _ { i = 1 } ^ { n } \left[\mu_{A_{i}}\left(\frac{\mu_{A_{i}}+\mu_{B_{i}}}{2 \mu_{B_{i}}}\right)^{n}+\right.\right.
$$




$$
\left.\left.\left(1-\mu_{A_{i}}\right)\left(\frac{2-\mu_{A_{i}}-\mu_{B_{i}}}{2\left(1-\mu_{B_{i}}\right)}\right)^{n}\right]-1\right)
$$

$n \neq 0,1$.

(vii) Fuzzy Hellinger Discrimination [1]:-

$$
\begin{aligned}
M(A, B) & =1-T(A, B) \\
& \left.=\frac{1}{2} \sum_{i=1}^{n}\left[\left(\sqrt{\mu_{A_{i}}}-\sqrt{\mu_{B_{i}}}\right)^{2}+\left(\sqrt{\left(1-\mu_{A_{i}}\right)}-\sqrt{\left(1-\mu_{B_{i}}\right.}\right)\right)^{2}\right],
\end{aligned}
$$

where $T(A, B)=\sum_{i=1}^{n}\left[\sqrt{\mu_{A_{i}} \mu_{B_{i}}}+\sqrt{\left(1-\mu_{B_{i}}\right)\left(1-\mu_{B_{i}}\right)}\right]$.

(viii) Fuzzy Triangular Discrimination [10]:-

$$
\triangle(A, B)=\sum_{i=1}^{n}\left[\frac{\left(\mu_{A_{i}}-\mu_{B_{i}}\right)^{2}}{\left(\mu_{A_{i}}+\mu_{B_{i}}\right)}+\frac{\left(\mu_{B_{i}}-\mu_{A_{i}}\right)^{2}}{\left(2-\mu_{A_{i}}-\mu_{B_{i}}\right)}\right],
$$

(ix) Fuzzy Symmetric Chi-Square Divergence [9]:-

$$
\begin{aligned}
\varphi(A, B) & =\chi^{2}(A, B)+\chi^{2}(B, A) \\
& =\sum_{i=1}^{n}\left[\frac{\left(\mu_{A_{i}}-\mu_{B_{i}}\right)^{2}\left(\mu_{A_{i}}+\mu_{B_{i}}\right)}{\left(\mu_{A_{i}} \mu_{B_{i}}\right)}+\frac{\left(\mu_{B_{i}}-\mu_{A_{i}}\right)^{2}\left(2-\mu_{A_{i}}-\mu_{B_{i}}\right)}{\left(1-\mu_{A_{i}}\right)\left(\left(1-\mu_{A_{i}}\right)\right.}\right]
\end{aligned}
$$

(x) Fuzzy Symmetric J-Divergence Measure [7]:-

$$
\begin{aligned}
N(A, B) & =K(A, B)+K(B, A) \\
& =\sum_{i=1}^{n}\left[\left(\mu_{A_{i}}-\mu_{B_{i}}\right) \log \left(\frac{\mu_{A_{i}}}{\mu_{B_{i}}}\right)+\left(\mu_{B_{i}}-\mu_{A_{i}}\right) \log \left(\frac{1-\mu_{A_{i}}}{1-\mu_{B_{i}}}\right)\right] .
\end{aligned}
$$

(xi) Fuzzy Relative J-Divergence Measure [29]:-

$$
\begin{aligned}
J_{m}(A, B)=\sum_{i=1}^{n}[ & \left(\mu_{A_{i}}-\mu_{B_{i}}\right) \log \left(\frac{\left(\mu_{A_{i}}+\mu_{B_{i}}\right)}{2 \mu_{B_{i}}}\right)+ \\
& \left.\left(\mu_{B_{i}}-\mu_{A_{i}}\right) \log \left(\frac{\left(2-\mu_{A_{i}}-\mu_{B_{i}}\right)}{2\left(1-\mu_{B_{i}}\right)}\right)\right] .
\end{aligned}
$$

(xii) Fuzzy Symmetric Arithmetic-Geometric Divergence [30] -

$$
\begin{gathered}
F(A, B)=\frac{1}{2}(H(A, B)+H(B, A))=\sum_{i=1}^{n}\left[\left(\frac{\mu_{A_{i}}+\mu_{B_{i}}}{2}\right) \log \left(\frac{\mu_{A_{i}}+\mu_{B_{i}}}{2 \sqrt{\mu_{A_{i}} \mu_{B_{i}}}}\right)+\right. \\
\left.\left(\frac{2-\mu_{A_{i}}-\mu_{B_{i}}}{2}\right) \log \left(\frac{2-\mu_{A_{i}}-\mu_{B_{i}}}{2 \sqrt{\left(1-\mu_{A_{i}}\right)\left(1-\mu_{B_{i}}\right)}}\right)\right]
\end{gathered}
$$


(xiii) Fuzzy Symmetric Chi-Square, Arithmetic and Geometric Mean Divergence Measure [17]:-

$$
E_{1}^{*}(A, B)=\sum_{i=1}^{n}\left[\left(\frac{\left(\mu_{A_{i}}-\mu_{B_{i}}\right)^{2}}{\sqrt{\mu_{A_{i}} \mu_{B_{i}}}}\right)+\left(\frac{\left(\mu_{B_{i}}-\mu_{A_{i}}\right)^{2}}{\sqrt{\left(1-\mu_{A_{i}}\right)\left(1-\mu_{B_{i}}\right)}}\right)\right]
$$

(xiv) Fuzzy Symmetric Jensen-Shannon Divergence Measure [5, 27]:-

$$
\begin{aligned}
& I(A, B)=S_{t}(A, B)+S_{t}(B, A) \\
& \quad=\frac{1}{2} \sum_{i=1}^{n}\left[\mu_{A_{i}} \log \left(\frac{2 \mu_{A_{i}}}{\mu_{A_{i}}+\mu_{B_{i}}}\right)+\mu_{B_{i}} \log \left(\frac{2 \mu_{B_{i}}}{\mu_{A_{i}}+\mu_{B_{i}}}\right)\right]+ \\
& \quad \frac{1}{2} \sum_{i=1}^{n}\left[\left(1-\mu_{A_{i}}\right) \log \left(\frac{2\left(1-\mu_{A_{i}}\right)}{2-\mu_{A_{i}}-\mu_{B_{i}}}\right)+\left(1-\mu_{B_{i}}\right) \log \left(\frac{2\left(1-\mu_{B_{i}}\right)}{2-\mu_{A_{i}}-\mu_{B_{i}}}\right)\right] .
\end{aligned}
$$

\section{New Fuzzy Information Inequality}

Theorem 3.1. Let $f: R_{+} \rightarrow R_{+}$be a convex function and normalized i.e. $f(1)=0$. Then $\forall A$ and $B$ be two fuzzy sets defined in universe of discourse $X=\left\{x_{1}, x_{2}, . ., x_{n}\right\}$ having the membership values $\mu_{A_{i}}$ and $\mu_{B_{i}}, i=1,2, . ., n$ respectively,

$$
\begin{aligned}
& \sum_{i=1}^{n}\left[\mu_{B_{i}} f\left(\frac{\mu_{A_{i}}+\mu_{B_{i}}}{2 \mu_{B_{i}}}\right)+\right.\left.\left(1-\mu_{B_{i}}\right) f\left(\frac{2-\mu_{A_{i}}-\mu_{B_{i}}}{2\left(1-\mu_{B_{i}}\right)}\right)\right] \\
& \leq \frac{1}{2} \sum_{i=1}^{n}\left[\mu_{B_{i}} f\left(\frac{\mu_{A_{i}}}{\mu_{B_{i}}}\right)+\left(1-\mu_{B_{i}}\right) f\left(\frac{1-\mu_{A_{i}}}{1-\mu_{B_{i}}}\right)\right], \\
& S_{f}(A, B) \leq \frac{1}{2} C_{f}(A, B),
\end{aligned}
$$

and equality holds in (3.1) iff $\frac{\mu_{A_{1}}}{\mu_{B_{1}}}=\frac{\mu_{A_{2}}}{\mu_{B_{2}}}=\cdots=\frac{\mu_{A_{n}}}{\mu_{B_{n}}}$.

Proof. Let $f: k \subset R \rightarrow R$ be differentiable convex on the interval $k$ and $y \in k^{0}$, where $k^{0}$ is interior of $k$. Let $\lambda=\left(\lambda_{1}, \lambda_{2}, \ldots ., \lambda_{n}\right) \in X_{n}$, then Jensen inequality is

$$
f\left(\sum_{i=n}^{n} \lambda_{i} y_{i}\right) \leq \sum_{i=n}^{n} \lambda_{i} f\left(y_{i}\right)
$$

if $\lambda_{1}=\lambda_{2}=\frac{1}{2}$ and $\lambda_{3}=\lambda_{4}=\cdots=\lambda_{n}=0$, then 


$$
f\left(\frac{y_{1}+y_{2}}{2}\right) \leq \frac{1}{2}\left[f\left(y_{1}\right)+f\left(y_{2}\right)\right]
$$

Putting $y_{1}=y$ and $y_{2}=1$ then

$$
f\left(\frac{y+1}{2}\right) \leq \frac{1}{2}[f(y)+f(1)] \leq \frac{1}{2} f(y)
$$

since $f(1)=0$. Put $y=\frac{\mu_{A_{i}}}{\mu_{B_{i}}}$ and apply fuzzy, then

$$
f\left(\frac{\mu_{A_{i}}+\mu_{B_{i}}}{2 \mu_{B_{i}}}\right)+f\left(\frac{2-\mu_{A_{i}}-\mu_{B_{i}}}{2\left(1-\mu_{B_{i}}\right)}\right) \leq \frac{1}{2}\left[f\left(\frac{\mu_{A_{i}}}{\mu_{B_{i}}}\right)+f\left(\frac{1-\mu_{A_{i}}}{1-\mu_{B_{i}}}\right)\right]
$$

Multiplying by $\mu_{B_{i}}$ and taking summation both side, we have

$$
\begin{aligned}
\sum_{i=1}^{n}\left[\mu_{B_{i}} f\left(\frac{\mu_{A_{i}}+\mu_{B_{i}}}{2 \mu_{B_{i}}}\right)+\right. & \left.\left(1-\mu_{B_{i}}\right) f\left(\frac{2-\mu_{A_{i}}-\mu_{B_{i}}}{2\left(1-\mu_{B_{i}}\right)}\right)\right] \\
\leq & \frac{1}{2} \sum_{i=1}^{n}\left[\mu_{B_{i}} f\left(\frac{\mu_{A_{i}}}{\mu_{B_{i}}}\right)+\left(1-\mu_{B_{i}}\right) f\left(\frac{1-\mu_{A_{i}}}{1-\mu_{B_{i}}}\right)\right]
\end{aligned}
$$

that is, $S_{f}(A, B) \leq \frac{1}{2} C_{f}(A, B)$. Equation (3.1) is a relation between fuzzy new $f$-divergence and fuzzy Csiszar $f$-divergence functional.

\section{Results Analysis:-}

In this section, we shall show relationship in the form of inequality using the results (3.1) of theorem (3.1) among the various known measures such as Csiszar's, new $f$-divergence, Hellinger, Chi-square, and J-divergence etc.

Result 1. Let $A, B \in X_{n}$ then we have the following relations

$$
\begin{aligned}
S_{t}(A, B) & \leq \frac{1}{2} K(A, B), \\
S_{t}(B, A) & \leq \frac{1}{2} K(B, A),
\end{aligned}
$$

where $S_{t}(A, B)$ and $K(A, B)$ are Fuzzy Relative Jensen Shannon Divergence Measure and Fuzzy K-L Divergence Measure given in (2.1) and (2.4).

Proof. Consider the mapping $f:(0, \infty) \longrightarrow R$,

$$
f(y)=-\log y, f^{\prime \prime}(y)=\frac{1}{y^{2}}>0 \forall y>0, f(1)=0,
$$


so $f$ is convex and normalized. Then

$$
\begin{array}{r}
S_{f}(A, B)=\sum_{i=1}^{n}\left[\mu_{B_{i}} f\left(\frac{\mu_{A_{i}}+\mu_{B_{i}}}{2 \mu_{B_{i}}}\right)+\left(1-\mu_{B_{i}}\right) f\left(\frac{2-\mu_{A_{i}}-\mu_{B_{i}}}{2\left(1-\mu_{B_{i}}\right)}\right)\right] \\
=\sum_{i=1}^{n}\left[\mu_{B_{i}} \log \left(\frac{2 \mu_{B_{i}}}{\mu_{A_{i}}+\mu_{B_{i}}}\right)+\left(1-\mu_{B_{i}}\right) \log \left(\frac{2\left(1-\mu_{B_{i}}\right)}{2-\mu_{A_{i}}-\mu_{B_{i}}}\right)\right]=S_{t}(B, A) .
\end{array}
$$

Interchange by $A \mapsto B$,

$$
\begin{array}{r}
S_{f}(B, A)=\sum_{i=1}^{n}\left[\mu_{A_{i}} f\left(\frac{\mu_{A_{i}}+\mu_{B_{i}}}{2 \mu_{A_{i}}}\right)+\left(1-\mu_{A_{i}}\right) f\left(\frac{2-\mu_{A_{i}}-\mu_{B_{i}}}{2\left(1-\mu_{A_{i}}\right)}\right)\right] \\
=\sum_{i=1}^{n}\left[\mu_{A_{i}} \log \left(\frac{2 \mu_{A_{i}}}{\mu_{B_{i}}+\mu_{A_{i}}}\right)+\left(1-\mu_{A_{i}}\right) \log \left(\frac{2\left(1-\mu_{A_{i}}\right)}{2-\mu_{B_{i}}-\mu_{A_{i}}}\right)\right]=S_{t}(A, B)
\end{array}
$$

Similarly,

$$
\begin{aligned}
C_{f}(A, B) & =\sum_{i=1}^{n}\left[\mu_{B_{i}} f\left(\frac{\mu_{A_{i}}}{\mu_{B_{i}}}\right)+\left(1-\mu_{B_{i}}\right) f\left(\frac{1-\mu_{A_{i}}}{1-\mu_{B_{i}}}\right)\right] \\
& =\sum_{i=1}^{n}\left[\mu_{B_{i}} \log \left(\frac{\mu_{B_{i}}}{\mu_{A_{i}}}\right)+\left(1-\mu_{B_{i}}\right) \log \left(\frac{1-\mu_{B_{i}}}{1-\mu_{A_{i}}}\right)\right]=K(B, A)
\end{aligned}
$$

Interchange by $A \mapsto B$,

$$
\begin{aligned}
C_{f}(B, A) & =\sum_{i=1}^{n}\left[\mu_{A_{i}} f\left(\frac{\mu_{B_{i}}}{\mu_{A_{i}}}\right)+\left(1-\mu_{A_{i}}\right) f\left(\frac{1-\mu_{B_{i}}}{1-\mu_{A_{i}}}\right)\right] \\
& =\sum_{i=1}^{n}\left[\mu_{A_{i}} \log \left(\frac{\mu_{A_{i}}}{\mu_{B_{i}}}\right)+\left(1-\mu_{A_{i}}\right) \log \left(\frac{1-\mu_{A_{i}}}{\left(1-\mu_{B_{i}}\right)}\right)\right]=K(A, B)
\end{aligned}
$$

From equations (3.1), (4.3), (4.4), (4.5) and (4.6) give the results (4.1) and (4.2).

Result 2. Consider $A, B \in X_{n}$, then we have the following relations

$$
H(B, A) \leq \frac{1}{2} K(A, B)
$$

where $K(A, B)$ and $H(A, B)$ are Fuzzy K-L divergence and Fuzzy Relative Arithmetic-Geometric divergence measure given in (2.1) and (2.5).

Proof. Consider the mapping $f:(0, \infty) \longrightarrow R$

$$
f(y)=y \log y, f^{\prime \prime}(y)=\frac{1}{y}>0, \forall y>0, f(1)=0,
$$


so $f$ is convex and normalized. Then

$$
\begin{aligned}
S_{f}(A, B) & =\sum_{i=1}^{n}\left[\mu_{B_{i}} f\left(\frac{\mu_{A_{i}}+\mu_{B_{i}}}{2 \mu_{B_{i}}}\right)+\left(1-\mu_{B_{i}}\right) f\left(\frac{2-\mu_{A_{i}}-\mu_{B_{i}}}{2\left(1-\mu_{B_{i}}\right)}\right)\right] \\
= & \sum_{i=1}^{n}\left[\left(\frac{\mu_{A_{i}}+\mu_{B_{i}}}{2}\right) \log \left(\frac{\mu_{A_{i}}+\mu_{B_{i}}}{2 \mu_{B_{i}}}\right)+\left(\frac{2-\mu_{A_{i}}-\mu_{B_{i}}}{2}\right) \log \left(\frac{2-\mu_{A_{i}}-\mu_{B_{i}}}{2\left(1-\mu_{B_{i}}\right)}\right)\right] \\
= & H(B, A) \\
C_{f}(A, B) & =\sum_{i=1}^{n}\left[\mu_{B_{i}} f\left(\frac{\mu_{A_{i}}}{\mu_{B_{i}}}\right)+\left(1-\mu_{B_{i}}\right) f\left(\frac{1-\mu_{A_{i}}}{1-\mu_{B_{i}}}\right)\right] \\
& =\sum_{i=1}^{n}\left[\mu_{A_{i}} \log \left(\frac{\mu_{A_{i}}}{\mu_{B_{i}}}\right)+\left(1-\mu_{A_{i}}\right) \log \left(\frac{1-\mu_{A_{i}}}{\left(1-\mu_{B_{i}}\right)}\right)\right]=K(A, B .)
\end{aligned}
$$

The equation (3.1) using (4.8) and (4.9) gives the result (4.7).

Result 3. Consider $A, B \in X_{n}$ then we have the following relations

$$
\begin{array}{r}
J_{m}(A, B) \leq N(A, B), \\
I(A, B)+F(A, B) \leq \frac{1}{2} N(A, B),
\end{array}
$$

where $N(A, B), J_{m}(A, B), F(A, B)$, and $I(A, B)$ are Fuzzy J-divergence, Fuzzy relative J-divergence, Fuzzy arithmetic-geometric divergence and Fuzzy jensen-Shannon divergence measure given in (2.10), (2.11),(2.12) and (2.14) respectively.

Proof. Consider the mapping $f:(0, \infty) \longrightarrow R$

$$
f(y)=(y-1) \log y, f^{\prime \prime}(y)=\frac{y+1}{y^{2}}>0, \forall y>0, f(1)=0,
$$

so $f$ is convex and normalized. Then

$$
\begin{aligned}
& S_{f}(A, B)=\sum_{i=1}^{n}\left[\mu_{B_{i}} f\left(\frac{\mu_{A_{i}}+\mu_{B_{i}}}{2 \mu_{B_{i}}}\right)+\left(1-\mu_{B_{i}}\right) f\left(\frac{2-\mu_{A_{i}}-\mu_{B_{i}}}{2\left(1-\mu_{B_{i}}\right)}\right)\right] \\
& =\frac{1}{2} \sum_{i=1}^{n}\left[\left(\mu_{A_{i}}-\mu_{B_{i}}\right) \log \left(\frac{\mu_{A_{i}}+\mu_{B_{i}}}{2 \mu_{B_{i}}}\right)+\left(\mu_{B_{i}}-\mu_{A_{i}}\right) \log \left(\frac{2-\mu_{A_{i}}-\mu_{B_{i}}}{2\left(1-\mu_{B_{i}}\right)}\right)\right] \\
& =\frac{1}{2} J_{m}(A, B) . \\
& C_{f}(A, B)=\sum_{i=1}^{n}\left[\mu_{B_{i}} f\left(\frac{\mu_{A_{i}}}{\mu_{B_{i}}}\right)+\left(1-\mu_{B_{i}}\right) f\left(\frac{1-\mu_{A_{i}}}{1-\mu_{B_{i}}}\right)\right]
\end{aligned}
$$




$$
=\sum_{i=1}^{n}\left[\left(\mu_{A_{i}}-\mu_{B_{i}}\right) \log \left(\frac{\mu_{A_{i}}}{\mu_{B_{i}}}\right)+\left(\mu_{B_{i}}-\mu_{A_{i}}\right) \log \left(\frac{1-\mu_{A_{i}}}{1-\mu_{B_{i}}}\right)\right]=N(A, B)
$$

The equation (3.1) using (4.12) and (4.13) gives (4.10).

Now again we get

$$
\begin{aligned}
& S_{f}(A, B) \\
& =\frac{1}{2} \sum_{i=1}^{n}\left[\mu_{A_{i}} \log \left(\frac{\mu_{A_{i}}+\mu_{B_{i}}}{2 \mu_{A_{i}}}\right)-\mu_{B_{i}} \log \left(\frac{\mu_{A_{i}} \mu_{B_{i}}}{2 \mu_{B_{i}}}\right)\right] \\
& \quad+\frac{1}{2} \sum_{i=1}^{n}\left[\left(1-\mu_{A_{i}}\right) \log \left(\frac{2-\mu_{A_{i}}-\mu_{B_{i}}}{2\left(1-\mu_{A_{i}}\right)}\right)-\left(1-\mu_{B_{i}}\right) \log \left(\frac{2-\mu_{A_{i}}-\mu_{B_{i}}}{2\left(1-\mu_{B_{i}}\right)}\right)\right] \\
& =\frac{1}{2} \sum_{i=1}^{n}\left[\mu_{A_{i}} \log \left(\frac{2 \mu_{A_{i}}}{\mu_{A_{i}}+\mu_{B_{i}}}\right)+\mu_{B_{i}} \log \left(\frac{2 \mu_{B_{i}}}{\left.\mu_{A_{i}}+\mu_{B_{i}}\right)}\right)\right] \\
& \quad+\frac{1}{2} \sum_{i=1}^{n}\left[\left(1-\mu_{A_{i}}\right) \log \left(\frac{2\left(1-\mu_{A_{i}}\right)}{2-\mu_{A_{i}}-\mu_{B_{i}}}\right)+\left(1-\mu_{B_{i}}\right) \log \left(\frac{2\left(1-\mu_{B_{i}}\right)}{2-\mu_{A_{i}}-\mu_{B_{i}}}\right)\right] \\
& \quad+\frac{1}{2} \sum_{i=1}^{n}\left[\mu_{A_{i}} \log \left(\frac{\left.\mu_{A_{i}}+\mu_{B_{i}} \frac{\mu_{A_{i}}+\mu_{B_{i}}}{2 \mu_{B_{i}}}\right)}{2 \mu_{A_{i}}}\right)\right. \\
& \left.\quad+\left(1-\mu_{B_{i}}\right) \log \left(\frac{\left(2-\mu_{A_{i}}-\mu_{B_{i}}\right)}{2\left(1-\mu_{B_{i}}\right)} \frac{\left(2-\mu_{A_{i}}-\mu_{B_{i}}\right)}{2\left(1-\mu_{A_{i}}\right)}\right)\right] \\
& =I(A, B)+\sum_{i=1}^{n}\left[\mu_{A_{i}} \log \left(\frac{\mu_{A_{i}}+\mu_{B_{i}}}{2 \sqrt{\mu_{A_{i}} \mu_{B_{i}}}}\right)+\left(1-\mu_{A_{i}}\right) \log \left(\frac{\left(2-\mu_{A_{i}}-\mu_{B_{i}}\right)}{2 \sqrt{\left(1-\mu_{A_{i}}\right)\left(1-\mu_{B_{i}}\right)}}\right)\right] .
\end{aligned}
$$

Using equations (3.1), (4.13) and (4.14), we get

$$
\begin{aligned}
I(A, B)+ & \sum_{i=1}^{n}\left[\mu_{A_{i}} \log \left(\frac{\mu_{A_{i}}+\mu_{B_{i}}}{2 \sqrt{\mu_{A_{i}} \mu_{B_{i}}}}\right)\right]+ \\
& \sum_{i=1}^{n}\left[\left(1-\mu_{A_{i}}\right) \log \left(\frac{2-\mu_{A_{i}}-\mu_{B_{i}}}{2 \sqrt{\left(1-\mu_{A_{i}}\right)\left(1-\mu_{B_{i}}\right)}}\right)\right] \leq \frac{1}{2} N(A, B)
\end{aligned}
$$

Interchanging $A$ and $B$ we get

$$
\begin{aligned}
I(B, A)+ & \sum_{i=1}^{n}\left[\mu_{B_{i}} \log \left(\frac{\mu_{A_{i}}+\mu_{B_{i}}}{2 \sqrt{\mu_{A_{i}} \mu_{B_{i}}}}\right)\right]+ \\
& \sum_{i=1}^{n}\left[\left(1-\mu_{B_{i}}\right) \log \left(\frac{2-\mu_{A_{i}}-\mu_{B_{i}}}{2 \sqrt{\left(1-\mu_{A_{i}}\right)\left(1-\mu_{B_{i}}\right)}}\right)\right] \leq \frac{1}{2} N(B, A) .
\end{aligned}
$$

Adding inequalities (4.15) and (4.16) 


$$
\begin{aligned}
& I(A, B)+I(B, A)+\sum_{i=1}^{n}\left[\left(\mu_{A_{i}}+\mu_{B_{i}}\right) \log \left(\frac{\mu_{A_{i}}+\mu_{B_{i}}}{2 \sqrt{\mu_{A_{i}} \mu_{B_{i}}}}\right)\right]+ \\
& \sum_{i=1}^{n}\left[\left(2-\mu_{A_{i}}-\mu_{B_{i}}\right) \log \left(\frac{2-\mu_{A_{i}}-\mu_{B_{i}}}{2 \sqrt{\left(1-\mu_{A_{i}}\right)\left(1-\mu_{B_{i}}\right)}}\right)\right] \leq \frac{1}{2} N(A, B)+\frac{1}{2} N(B, A) \\
& 2 I(A, B)+2 F(A, B) \leq \\
& \sum_{i=1}^{n}\left[\left(\mu_{A_{i}}-\mu_{B_{i}}\right) \log \left(\frac{\mu_{A_{i}}}{\mu_{B_{i}}}\right)\right]+\sum_{i=1}^{n}\left[\left(\mu_{B_{i}}-\mu_{A_{i}}\right) \log \left(\frac{\left(1-\mu_{A_{i}}\right)}{\left(1-\mu_{B_{i}}\right.}\right)\right]
\end{aligned}
$$

Therefore $I(A, B)=I(B, A)$ and $2 I(A, B)+2 F(A, B) \leq N(A, B)$.

Note that, from equation (4.11), we get

$\left(\mathrm{a}_{1}\right) I(A, B) \leq \frac{1}{2} N(A, B)$

$\left(\mathrm{a}_{2}\right) F(A, B) \leq \frac{1}{2} N(A, B)$, with equality if $A=B$, where $I(A, B)$ and $F(A, B)$ both are positive.

Result 4. Let $A, B \in X_{n}$ then we have the following relations

$$
\triangle(A, B)<E_{1}^{*}(A, B)
$$

where $\triangle(A, B)$ and $E_{1}^{*}(A, B)$ are Fuzzy Triangular discrimination and Fuzzy Symmetric Chi square, Arithmetic and Geometric mean divergence measure give in (2.8) and (2.13) respectively.

Proof. Consider the mapping $f:(0, \infty) \longrightarrow R$

$$
f(y)=\frac{(y-1)^{2}}{\sqrt{y}}, f^{\prime \prime}(y)=\frac{3}{4} y^{\frac{-1}{2}}+\frac{3}{4} y^{\frac{-5}{2}}+\frac{1}{2} y^{\frac{-3}{2}}>0, \forall y>0, f(1)=0,
$$

so $f$ is convex and normalized. Then

$$
\begin{aligned}
S_{f}(A, B) & =\sum_{i=1}^{n}\left[\mu_{B_{i}} f\left(\frac{\mu_{A_{i}}+\mu_{B_{i}}}{2 \mu_{B_{i}}}\right)+\left(1-\mu_{B_{i}}\right) f\left(\frac{2-\mu_{A_{i}}-\mu_{B_{i}}}{2\left(1-\mu_{B_{i}}\right)}\right)\right] \\
& =\sum_{i=1}^{n}\left[\left(\frac{\left(\mu_{A_{i}}-\mu_{B_{i}}\right)^{2}}{\sqrt{2 \mu_{B_{i}}\left(\mu_{A_{i}}+\mu_{B_{i}}\right)}}\right)+\left(\frac{\left(\mu_{B_{i}}-\mu_{A_{i}}\right)^{2}}{\sqrt{2\left(1-\mu_{B_{i}}\right)\left(2-\mu_{B_{i}}-\mu_{A_{i}}\right)}}\right)\right], \\
C_{f}(A, B) & =\sum_{i=1}^{n}\left[\mu_{B_{i}} f\left(\frac{\mu_{A_{i}}}{\mu_{B_{i}}}\right)+\left(1-\mu_{B_{i}}\right) f\left(\frac{1-\mu_{A_{i}}}{1-\mu_{B_{i}}}\right)\right]
\end{aligned}
$$




$$
=\sum_{i=1}^{n}\left[\left(\frac{\left(\mu_{A_{i}}-\mu_{B_{i}}\right)^{2}}{\sqrt{\mu_{A_{i}} \mu_{B_{i}}}}\right)+\left(\frac{\left(\mu_{B_{i}}-\mu_{A_{i}}\right)^{2}}{\sqrt{\left(1-\mu_{A_{i}}\right)\left(1-\mu_{B_{i}}\right)}}\right)\right]=E_{1}^{*}(A, B) .
$$

From equations (3.1), (4.18) and (4.19)

$$
\begin{aligned}
\sum_{i=1}^{n}\left[E_{1}^{*}(A, B)\right. & \left.\geq 2\left(\frac{\left(\mu_{A_{i}}-\mu_{B_{i}}\right)^{2}}{\sqrt{2 \mu_{B_{i}}\left(\mu_{A_{i}}+\mu_{B_{i}}\right)}}\right)+\left(\frac{\left(\mu_{B_{i}}-\mu_{A_{i}}\right)^{2}}{\sqrt{2\left(1-\mu_{B_{i}}\right)\left(2-\mu_{B_{i}}-\mu_{A_{i}}\right)}}\right)\right] \\
& \left.=\sqrt{2}\left(\frac{\left(\mu_{A_{i}}-\mu_{B_{i}}\right)^{2}}{\sqrt{\mu_{B_{i}}\left(\mu_{A_{i}}+\mu_{B_{i}}\right)}}\right)+\left(\frac{\left(\mu_{B_{i}}-\mu_{A_{i}}\right)^{2}}{\sqrt{\left(1-\mu_{B_{i}}\right)\left(2-\mu_{B_{i}}-\mu_{A_{i}}\right)}}\right)\right],
\end{aligned}
$$

and

$$
\begin{aligned}
& \triangle(A, B)= \sum_{i=1}^{n}\left[\left(\frac{\left(\mu_{A_{i}}-\mu_{B_{i}}\right)^{2}}{\left(\mu_{A_{i}}+\mu_{B_{i}}\right)}\right)+\left(\frac{\left(\mu_{B_{i}}-\mu_{A_{i}}\right)^{2}}{\left(2-\mu_{A_{i}}-\mu_{B_{i}}\right)}\right)\right] \\
&<\sqrt{2} \sum_{i=1}^{n}\left[\left(\frac{\left(\mu_{A_{i}}-\mu_{B_{i}}\right)^{2}}{\sqrt{\left(\mu_{A_{i}}\right)^{2}+\left(\mu_{B_{i}}\right)^{2}+2 \mu_{A_{i}} \mu_{B_{i}}}}\right)+\right. \\
& \\
&\left.\quad\left(\frac{\left(\mu_{B_{i}}-\mu_{A_{i}}\right)^{2}}{\sqrt{\left(1-\mu_{A_{i}}\right)^{2}+\left(1-\mu_{B_{i}}\right)^{2}+2\left(1-\mu_{A_{i}}\right)\left(1-\mu_{B_{i}}\right)}}\right)\right] \\
&<\sqrt{2} \sum_{i=1}^{n}\left[\left(\frac{\left(\mu_{A_{i}}-\mu_{B_{i}}\right)^{2}}{\sqrt{\mu_{B_{i}}\left(\mu_{A_{i}}+\mu_{B_{i}}\right)}}\right)+\left(\frac{\left(\mu_{B_{i}}-\mu_{A_{i}}\right)^{2}}{\sqrt{\left(1-\mu_{B_{i}}\right)\left(2-\mu_{A_{i}}-\mu_{B_{i}}\right)}}\right)\right] \\
& \leq E_{1}^{*}(A, B) .
\end{aligned}
$$

Hence the relation (4.17) is proved.

Result 5. Let $A, B \in X_{n}$ then we have the following relations

$$
\frac{1}{2} \chi^{2}(A, B)+\triangle(A, B) \leq \varphi(A, B)
$$

where $\chi^{2}(A, B), \triangle(A, B)$ and $\varphi(A, B)$ are fuzzy Chi-square divergence, Fuzzy triangular discrimination and Fuzzy symmetric Chi-square divergence measure give in (2.3), (2.8) and (2.9) respectively.

Proof. Consider the mapping $f:(0, \infty) \longrightarrow R$

$$
f(y)=\frac{(y-1)^{2}(y+1)}{y}, f^{\prime \prime}(y)=2+\frac{2}{y^{3}}>0, \forall y>0, f(1)=0,
$$

so $f$ is convex and normalized. Then

$$
S_{f}(A, B)=\sum_{i=1}^{n}\left[\mu_{B_{i}} f\left(\frac{\mu_{A_{i}}+\mu_{B_{i}}}{2 \mu_{B_{i}}}\right)+\left(1-\mu_{B_{i}}\right) f\left(\frac{2-\mu_{A_{i}}-\mu_{B_{i}}}{2\left(1-\mu_{B_{i}}\right)}\right)\right]
$$




$$
\begin{aligned}
= & \frac{1}{4} \sum_{i=1}^{n}\left[\frac{\left(\mu_{A_{i}}-\mu_{B_{i}}\right)^{2}}{\mu_{B_{i}}}+\frac{\left(\mu_{A_{i}}-\mu_{B_{i}}\right)^{2}}{\left(1-\mu_{B_{i}}\right)}\right]+\frac{1}{2} \sum_{i=1}^{n}\left[\frac{\left(\mu_{A_{i}}-\mu_{B_{i}}\right)^{2}}{\mu_{A_{i}}+\mu_{B_{i}}}+\frac{\left(\mu_{A_{i}}-\mu_{B_{i}}\right)^{2}}{2-\mu_{A_{i}}-\mu_{B_{i}}}\right] \\
= & \frac{1}{4} \chi^{2}(A, B)+\frac{1}{2} \triangle(A, B), \\
& C_{f}(A, B)=\sum_{i=1}^{n}\left[\mu_{B_{i}} f\left(\frac{\mu_{A_{i}}}{\mu_{B_{i}}}\right)+\left(1-\mu_{B_{i}}\right) f\left(\frac{1-\mu_{A_{i}}}{1-\mu_{B_{i}}}\right)\right]=\varphi(A, B)
\end{aligned}
$$

The equation (3.1) using (4.21) and (4.22) gives the result (4.20).

Result 6. Let $A, B \in X_{n}$ then we have the following relations

$$
M G_{n}(B, A) \leq \frac{1}{2} D_{n}(A, B)
$$

where $M G_{n}(A, B)$ and $D_{n}(A, B)$ are Unified fuzzy relative Jensen-Shannon and ArithmeticGeometric Divergence of type $n$ and Fuzzy relative information of type $n$ give in (2.6) and (2.2), respectively.

Proof. Consider the mapping $f:(0, \infty) \longrightarrow R$

$$
f_{n}(y)= \begin{cases}{[n(n-1)]^{-1}\left[y^{n}-1\right]} & \text { if } \neq 0,1 \\ -\log y & \text { if } n=0 \\ y \log y & \text { if } n=1\end{cases}
$$

Since $f^{\prime \prime}(y) \geq 0$ and $f(1)=0$, so the function $f$ is convex and normalized. Then

$$
\begin{aligned}
& S_{f}(A, B)=\sum_{i=1}^{n}\left[\mu_{B_{i}} f\left(\frac{\mu_{A_{i}}+\mu_{B_{i}}}{2 \mu_{B_{i}}}\right)+\left(1-\mu_{B_{i}}\right) f\left(\frac{2-\mu_{A_{i}}-\mu_{B_{i}}}{2\left(1-\mu_{B_{i}}\right)}\right)\right] \\
& =[n(n-1)]^{-1}\left[\left[\sum_{i=1}^{n} \mu_{B_{i}}\left(\frac{\mu_{A_{i}}+\mu_{B_{i}}}{2 \mu_{B_{i}}}\right)^{n}+\left(1-\mu_{B_{i}}\right)\left(\frac{2-\mu_{A_{i}}-\mu_{B_{i}}}{2\left(1-\mu_{B_{i}}\right)}\right)^{n}\right]-1\right] \\
& =M G_{n}(B, A)
\end{aligned}
$$

$$
\begin{aligned}
& C_{f}(A, B)=\sum_{i=1}^{n}\left[\mu_{B_{i}} f\left(\frac{\mu_{A_{i}}}{\mu_{B_{i}}}\right)+\left(1-\mu_{B_{i}}\right) f\left(\frac{1-\mu_{A_{i}}}{1-\mu_{B_{i}}}\right)\right] \\
& \quad=(n(n-1))^{-1}\left[\sum_{i=1}^{n}\left(\left(\mu_{A_{i}}\right)^{n}\left(\mu_{B_{i}}\right)^{1-n}+\left(1-\mu_{A_{i}}\right)^{n}\left(1-\mu_{B_{i}}\right)^{1-n}\right)-1\right]
\end{aligned}
$$




$$
=D_{n}(A, B)
$$

The quation (3.1) using (4.24), (4.25) and (4.26) gives the result (4.23).

\section{Particular Cases of Result 4.6:-}

(i) If $n=0,1$ then we have proved in equation (4.1) and (4.7) of results in (4.1) and (4.2) respectively.

(ii) If $n=-1$ then we get the following relation

$$
\triangle(A, B) \leq \chi^{2}(B, A)
$$

Proof. Put $n=-1$ in equation (4.23), then

$$
\begin{gathered}
M G_{-1}(B, A) \leq \frac{1}{2} D_{-1}(A, B) \\
M G_{-1}(B, A)=\frac{1}{2}\left(\sum_{i=1}^{n}\left[\frac{2\left(\mu_{B_{i}}\right)^{2}}{\mu_{A_{i}}+\mu_{B_{i}}}+\frac{2\left(1-\mu_{B_{i}}\right)^{2}}{2-\mu_{A_{i}}-\mu_{B_{i}}}\right]-1\right) \\
=\frac{1}{2}\left(\sum_{i=1}^{n}\left[2 \mu_{B_{i}}-\frac{2 \mu_{A_{i}} \mu_{B_{i}}}{\mu_{A_{i}}+\mu_{B_{i}}}+2\left(1-\mu_{B_{i}}\right)-\frac{2\left(1-\mu_{A_{i}}\right)\left(1-\mu_{B_{i}}\right)}{2-\mu_{A_{i}}-\mu_{B_{i}}}\right]-1\right) \\
=\frac{1}{2}\left(2-\sum_{i=1}^{n}\left[\frac{2 \mu_{A_{i}} \mu_{B_{i}}}{\mu_{A_{i}}+\mu_{B_{i}}}+\frac{2\left(1-\mu_{A_{i}}\right)\left(1-\mu_{B_{i}}\right)}{2-\mu_{A_{i}}-\mu_{B_{i}}}\right]-1\right) \\
=\frac{1}{2}\left[1-\sum_{i=1}^{n}\left(\frac{\left(2 \mu_{A_{i}} \mu_{B_{i}}\right)}{\left(\mu_{A_{i}}+\mu_{B_{i}}\right)}+\frac{2\left(1-\mu_{A_{i}}\right)\left(1-\mu_{B_{i}}\right)}{\left(2-\mu_{A_{i}}-\mu_{B_{i}}\right)}\right)\right] \\
\\
M G_{-1}(B, A)=\frac{1}{4} \triangle(A, B)[\mathrm{using}(2.8) \text { and }(4.25)]
\end{gathered}
$$

and

$$
D_{-1}(A, B)=\frac{1}{2} \chi^{2}(B, A)[\operatorname{using}(2.3)(2.3) \text { and }(4.26)]
$$

using equations (4.28), (4.29) and (4.30) gives the result (4.27).

(iii) if $n=\frac{1}{2}$, then we have the following relation 


$$
4\left[1-T\left(\frac{A+B}{2}, B\right)\right] \leq M(A, B) .
$$

Proof. If $n=\frac{1}{2}$ put in (4.23)

$$
M G_{\frac{1}{2}}(B, A) \leq \frac{1}{2} D_{\frac{1}{2}}(A, B)
$$

where

$$
\begin{aligned}
M G_{\frac{1}{2}}(B, A) & =4\left[1-\sum_{i=1}^{n}\left(\frac{\sqrt{\mu_{A_{i}} \mu_{B_{i}}+\left(\mu_{B_{i}}\right)^{2}}}{2}+\frac{\sqrt{\left(1-\mu_{A_{i}}\right)\left(1-\mu_{B_{i}}\right)+\left(1-\mu_{B_{i}}\right)^{2}}}{2}\right)\right] \\
& =4\left[1-T\left(\frac{A+B}{2}, B\right)\right]
\end{aligned}
$$

(using (2.7) and (4.25)) and

$$
D_{\frac{1}{2}}(A, B)=4\left[1-\sum_{i=1}^{n}\left(\sqrt{\mu_{A_{i}}} \mu_{B_{i}}+\sqrt{\left(1-\mu_{A_{i}}\right)\left(1-\mu_{B_{i}}\right.}\right)\right]=2 M(A, B) .
$$

using equations (4.32), (4.33) and (4.34) gives the result (4.31).

\section{Case Study}

We consider the problem having four known patterns $A_{1}, A_{2}, A_{3}$ and $A_{4}$ which have classifications $R_{1}, R_{2}, R_{3}$ and $R_{4}$ respectively. These are represented by the following fuzzy sets in the universe of discourse $X=\left\{x_{1}, x_{2}, x_{3}\right\}$.

$$
\begin{aligned}
& A_{1}=\left\{<x_{1}, .7>,<x_{2}, .3>,<x_{3}, .1>\right\}, \\
& A_{2}=\left\{<x_{1}, .4>,<x_{2}, .2>,<x_{3}, .5>\right\}, \\
& A_{3}=\left\{<x_{1}, .6>,<x_{2}, .3>,<x_{3}, .8>\right\}, \\
& A_{4}=\left\{<x_{1}, .7>,<x_{2}, .5>,<x_{3}, .8>\right\} .
\end{aligned}
$$

Given an unknown pattern $B$, represented by the fuzzy set

$$
B=\left\{<x_{1}, .5>,<x_{2}, .4>,<x_{3}, .9>\right\}
$$

From the calculated numerical values of fuzzy divergence measure given in Table 1, the inequalities proposed in result (4.1) are verified

$$
S_{t}(A, B) \leq \frac{1}{2} K(A, B) .
$$


Table 1: Calculated numerical values of fuzzy divergence measures.

\begin{tabular}{|c|c|}
\hline $\mathrm{S}_{t}(\mathrm{~A}, \mathrm{~B})$ & $\mathrm{K}(\mathrm{A}, \mathrm{B})$ \\
\hline .1717 & .8085 \\
\hline .0512 & .2703 \\
\hline $\mathbf{. 0 0 8 5}$ & $\mathbf{. 0 3 7 4}$ \\
\hline .0154 & .0639 \\
\hline
\end{tabular}

\section{Conclusions}

In this current paper, we have achieved some series of fuzzy divergence measure by using fuzzy Csiszar's $f$-divergence and fuzzy new $f$-divergence measures properties with proof of validity, We have recommended generalized series of combination of kullback-Leibler, Arithmetic divergence measure etc. we have also derived Inequalities relating with new and well known fuzzy divergence measures.

\section{Acknowledgments}

We would like to thank you for following the instructions above very closely in advance. It will definitely save us lot of time and expedite the process of your paper's publication.

\section{References}

[1] R. Beran , Minimum Hellinger distance estimates for parametric models Ann.Statist.5 (1977), 445-463.

[2] D. Bhandari and N. R Pal, Some new information measures for fuzzy sets. Information Sciences, 67(3), (1993), 209-228.

[3] A. Bhattacharyya, Some Analogues to the Amount of Information and Their uses in Statistical Estimation, Sankhya, 8(1946), 1-14.

[4] P. K. Bhatia and S. Singh, Three families of generalized fuzzy directed divergence, AMOAdvanced Modeling and Optimization, 14(3) (2012), 599-614.

[5] J. Burbea, and C. R. Rao, On Convexity of Some Divergence measures based on entropy functions,IEEE Transe.on.inform.theory ,IT-28 (1982),489-495. 
[6] I. Csisza, On Topological Properties of $f$-Divergences. Studia Math. Hungarica, Vol. 2 (1967), 329-339.

[7] I. Csiszar, Information-type measures of difference of probability functions and indirect observations. Studia Sci. Math. Hunger. 2(1961). 299-318

[8] Wu Dongrui and Jerry M. Mendel, Uncertainty measures for interval type-2 fuzzy sets, Information Sciences: an International Journal, v.177 n.23 (2007),p.5378-5393.

[9] S. S. Dragomir, J. Sunde and C. Buse, New Inequalities for Jeffreys Divergence measure, Tamusi Oxford Journal of Mathematical Sciences,16(2)(2000),295-309.

[10] S. S. Dragomir, Bounds of $f$-divergences under likelihood Ratio Constraints No.3 (2003), 205-223.

[11] M. Ghosh, D. Das, C. Chakraborty and A. K. Roy, Automated leukocyte recognition using fuzzy divergence, Micron, 41 (2010), 840-846.

[12] D. S. Hooda and D. Jain, The generalized fuzzy measures of directed divergence, total ambiguity and information improvement, Investigations in Mathematical Sciences, 2 (2012), 239-260.

[13] E. Hellinger and Neue Begr undung der Theorie der quadratischen Formen von unendlichen vielen Ver anderlichen, J. Reine Aug. Math., 136(1909), 210-271

[14] K. C. Jain and R. N. Saraswat, New Information Inequality and its application in establishing relation among various $f$-divergence measures, Journal of the Applied Mathematics, Statistics and Informatics (JAMSI), 8 (2012).

[15] K. C. Jain and R. N. Saraswat Some well-known inequalities and its applications in information theory" Jordan Journal of Mathematics and Statistics. 2013, 157-167

[16] K. C. Jain and R. N. Saraswat Some Bounds of Information Divergence Measures in Terms of Relative-Arithmetic Divergence Measure” International Journal of Applied Mathematics and Statistics, 32 (2) (2013), 48-58.

[17] K. C. Jain and A. Srivastava, On Symmetric Information Divergence Measures of Csiszar's $f$-Divergence Class, Journal of Applied Mathematics, Statistics and Informatics (JAMSI),3(1)(2007),85-102

[18] A. Kaufmann, Introduction to the Theory of Fuzzy Sets Fundamental Theoretical Elements. 1975. Academic Press, New York, v1. 
[19] S. Kullback and R. A. Leibler, On information and sufficiency, The Annals of Mathematical Statistics 22(1) (1951), 79-86.

[20] A. Luca De. and S. Termini, A definition of non-probabilistic entropy in the setting of fuzzy sets theory. Information and Control. v20 (1972), 301-312.

[21] Loo and S. G., Measures of fuzziness. Cybernetica. v20 (1997), 201-210.

[22] S. Montes, I. Couso, P. Gil and C. Bertoluzza, Divergence measures between fuzzy sets, International Journal of Approximate Reasoning, 30 (2002) 91-105.

[23] O. Parkash, P. K. Sharma and S. Kumar, Two new measures of fuzzy divergence and their properties. SQU Journal for Science, 11 (2006) 69-77.

[24] K. Pearson, On the criterion that a give system of deviations from the probable in the case of correlated system of variables in such that it can be reasonable supposed to have arisen from random sampling, Phil. Mag., 50(1900),157-172.

[25] F. Osterreicher, Csiszar's $f$-Divergence, Basic Properties, pre-print, 2002.

[26] R. N. Saraswat and Ajay Tak, New $f$-divergence and jensen-ostrowski's type inequalities, Tamkang Journal of Mathematics 50(2019), 111-118.

[27] R. Sibson, Information Radius,Z,Wahrs.undverw.geb.,14(1969),149-160.

[28] C. E. Shannon, A mathematical theory of communication, The Bell Syst. Tech. Journal 27(3) (1948) 379-423.

[29] I. J. Taneja and Prenesh Kumar, Relative information of type s, Csiszar's $f$-divergence, and information inequalities, Information sciences 166 (2004) 105-125

[30] I. J. Taneja, New Developments in generalized information measures, Chapter in: Advances in imaging and Electron Physics, Ed. P.W. Hawkes 91 (1995),37-135.

[31] I. J. Taneja, Pranesh Kumar, Generalized non-symmetric divergence measures and inequalities.

[32] V. P. Tomar and A. Ohlan, Sequence of inequalities among fuzzy mean difference divergence measures and their applications, Springer Plus, 3: 623 (2014), 1-20.

[33] F. Topse, Some inequalities for information divergence and related measures of discrimination.Res.Coll.RGMIA2 (1999), 85-98.. 
[34] L. A. Zadeh, Probability measures of fuzzy events. Journal of Mathematical Analysis and Applications. v23 (1965), 421-427.

[35] L. A. Zadeh, Toward a generalized theory of uncertainty (GTU): an outline, Information Sciences-Informatics and Computer Science: An International Journal, v.172 n.1-2 (2005), p.1-40.

Sapna Gahlot Department of Mathematics and Statistics, Manipal University Jaipur Jaipur( Rajasthan) - 303007, INDIA

E-mail: sapna1994gahlot@gmail.com

Ram Naresh Saraswat Department of Mathematics and Statistics, Manipal University Jaipur, Jaipur( Rajasthan) - 303007, INDIA

E-mail: saraswatramn@gmail.com 